\title{
Design of fault detection filters for periodic systems
}

\author{
A. Varga
}

\begin{abstract}
We propose a numerically reliable computational approach to design fault detection filters for periodic systems. This approach is based on a new numerically stable algorithm to compute least order annihilators without explicitly building time-invariant lifted system representations. The main computation in this algorithm is the orthogonal reduction of a periodic matrix pair to a periodic Kronecker-like form, from which the periodic realization of the detector is directly obtained.
\end{abstract}

\section{INTRODUCTION}

We develop a design procedure of residual generators for periodic systems which provide two basic functions: (1) generate zero residuals in the fault-free case; (2) generate nonzero residuals when any fault occurs in the system. A more advanced functionality, like fault isolation (i.e., exact localization of faults) can be often achieved by designing a bank of such fault detectors [1]. The solution of the periodic fault detection problem has its main application in solving multirate fault detection problems in the most general setting.

The fault detection problem for linear multirate sampleddata systems has been addressed recently by Fadali [2] and by Zhang et al. [3]. The proposed solutions rely on explicitly building time-invariant lifted representations of the underlying multirate systems and employing design techniques developed for standard linear systems. Although such an approach can be easily extended to the general periodic case, still there are several difficulties which can impede his usage for systems with high orders or large periods. For example, building a lifted representation using the lifting technique of [4] involves explicitly forming many matrix products, thus this approach is completely unappropriate from numerical point of view. On the other hand, using the lifting technique proposed in [5] requires manipulating large sparse matrices of a descriptor system representation, which leads to computationally unacceptable costs. Even the final step of turning the designed lifted representation of the detector into a periodic state space representation (e.g., by using the algorithm of [6]) can lead to numerical difficulties in the case of high order systems.

Recently, the fault detection problem with $\mathcal{H}_{\infty}$-optimal disturbance attenuation has been considered for periodic discrete-time systems [7] and a solution approach has been proposed without employing lifting. Avoiding the above mentioned difficulties related to lifting was also our main motivation to investigate an alternative approach to design

A. Varga is with the German Aerospace Center, DLR - Oberpfaffenhofen, Institute of Robotics and Mechatronics, D-82234 Wessling, Germany; Email: andras.varga@dlr.de periodic fault detectors directly in a minimal periodic statespace representation. The proposed approach (see Section II) is a generalization of the method proposed by the author in [8] and involves, as main computational step, the determination of a left annihilator of a certain periodic system. For this computation we developed a numerically stable algorithm to compute left annihilators for periodic systems (see Section III). The main computation in this algorithm is the orthogonal reduction of a periodic matrix pair to a Kronecker-like form, which allows to obtain, practically without any additional computation, a left annihilator. A stable left annihilator can be obtained by solving additionally a periodic stabilization or pole assignment problem.

We also develop easy to perform numerical tests to check the existence of a solution, and indicate a possible approach to solve the more involved fault isolation problem for periodic systems. Finally we discuss shortly a possible approach based on frequency-weighted balanced truncation to reduce the order of the detectors to allow an efficient implementation and operation of the residual generator.

Notation. For an $N$-periodic matrix $X_{i}$ we use systematically the script notation $\mathcal{X}:=\operatorname{diag}\left(X_{1}, X_{2}, \ldots, X_{N}\right)$, which associates the block-diagonal matrix $\mathcal{X}$ to the cyclic matrix sequence $X_{i}, i=1, \ldots, N$.

\section{Periodic Fault Detection Problems}

We consider periodic time-varying linear discrete-time systems of the form

$$
\begin{aligned}
E_{k} x(k+1) & =A_{k} x(k)+B_{k}^{u} u(k)+B_{k}^{d} d(k)+B_{k}^{f} f(k) \\
y(k) & =C_{k} x(k)+D_{k}^{u} u(k)+D_{k}^{d} d(k)+D_{k}^{f} f(k)
\end{aligned}
$$

where $x(k) \in \mathrm{R}^{n_{k}}$ is the system state vector with timevarying dimensions, $y(k)$ is the $p$-dimensional measurement output vector, $u(k)$ is the $m_{u}$-dimensional plant control input vector, $f(k)$ is the $m_{f}$-dimensional fault signal vector, and $d(k)$ is the $m_{d}$-dimensional disturbance vector. We assume that the system matrices are periodic with period $N \geq 1$ and $E_{k}$ are square and invertible for $k=1, \ldots, N$. The periodic fault detection problem (PFDP) for linear periodic discrete-time system can be formulated as follows:

Periodic Fault Detection Problem: Determine a periodic linear residual generator (or detector) having the general form

$$
\begin{aligned}
\widehat{x}(k+1) & =F_{k} \widehat{x}(k)+H_{k}^{y} y(k)+H_{k}^{u} u(k) \\
r(k) & =M_{k} x(k)+L_{k}^{y} y(k)+L_{k}^{u} u(k)
\end{aligned}
$$

such that for $k \geq 0$

(i) $r(k)=0$ if $f(k)=0$ (fault-free case);

(ii) $r(k) \neq 0$ if $f_{i}(k) \neq 0$, for $i=1, \ldots, m_{f}$

(fault detectability), 
where for both (i) and (ii) we assume zero initial conditions for the state variables (i.e., $x(0)=0$ and $\widehat{x}(0)=0$ ).

The condition $(i i)$ is also known as the weak fault detectability condition (see [9]), and covers the most general case of non-zero residual signals in the faulty case, regardless when they asymptotically vanish or not. However, in practice we are often interested to obtain non-zero steady state values of residual signals, when abrupt, but constant fault signals enter the system. The corresponding notion of strong fault detectability thus requires

$\left(i i^{\prime}\right) r(k) \neq 0$ if $f_{i}(k) \neq 0, i=1, \ldots, m_{f}$, for $k \rightarrow \infty$.

We call the corresponding problem the periodic strong fault detection problem (PSFDP). By solving PSFDP we solve also PFDP, but the converse is evidently not true.

To derive algebraic conditions for the solution of PFDP, we reformulate the detector design problem in terms of the transfer-function matrix (TFM) corresponding to the associated stacked lifted representation of [5], which uses the input-state-output behavior of the system over time intervals of length $N$, rather then 1 . The lifted input, output and state vectors are defined as

$$
\begin{aligned}
\widetilde{u}(h) & =\left[u^{T}(h N+1) \cdots u^{T}(h N+N)\right]^{T}, \\
\widetilde{d}(h) & =\left[d^{T}(h N+1) \cdots d^{T}(h N+N)\right]^{T}, \\
\widetilde{f}(h) & =\left[f^{T}(h N+1) \cdots f^{T}(h N+N)\right]^{T}, \\
\widetilde{y}(h) & =\left[y^{T}(h N+1) \cdots y^{T}(h N+N)\right]^{T}, \\
\widetilde{x}(h) & =\left[x^{T}(h N+1) \cdots x^{T}(h N+N)\right]^{T} .
\end{aligned}
$$

and the corresponding lifted system can be represented by a time-invariant descriptor system of the form (notice the usage of script notation)

$$
\begin{aligned}
\widetilde{E} \widetilde{x}(h+1) & =\widetilde{A} \widetilde{x}(h)+\mathcal{B}^{u} \widetilde{u}(h)+\mathcal{B}^{d} \widetilde{d}(h)+\mathcal{B}^{f} \widetilde{f}(h) \\
\widetilde{y}(h) & =\mathcal{C} \widetilde{x}(h)+\mathcal{D}^{u} \widetilde{u}(h)+\mathcal{D}^{d} \widetilde{d}(h)+\mathcal{D}^{f} \widetilde{f}(h)
\end{aligned}
$$

where the pole pencil corresponding to the periodic pair $\left(A_{k}, E_{k}\right)$

$$
\widetilde{A}-z \widetilde{E}=\left[\begin{array}{ccccc}
A_{1} & -E_{1} & O & \cdots & O \\
O & \ddots & \ddots & \ddots & \vdots \\
\vdots & \ddots & \ddots & -E_{N-2} & O \\
O & & \ddots & A_{N-1} & -E_{N-1} \\
-z E_{N} & O & \cdots & O & A_{N}
\end{array}\right]
$$

is regular. For the lifted system the TFMs $G_{u}(z), G_{d}(z)$, $G_{f}(z)$ from the control, disturbance, and fault inputs, respectively, to the system output are

$$
G_{x}(z)=\mathcal{C}(z \widetilde{E}-\widetilde{A})^{-1} \mathcal{B}^{x}+\mathcal{D}^{x}
$$

where $x$ stays for $u, d$ or $f$. Let denote by $G_{f}^{(i)}(z)$ the TFM of the lifted system corresponding to the $i$-th fault defined as

$$
G_{f}^{(i)}(z)=\mathcal{C}(z \widetilde{E}-\widetilde{A})^{-1} \mathcal{B}^{f, i}+\mathcal{D}^{f, i}
$$

where $B_{k}^{f, i}$ and $D_{k}^{f, i}$ are the $i$-th columns of matrices $B_{k}^{f}$ and $D_{k}^{f}$, respectively.
Assume that the linear residual generator (2) has a lifted representation with the corresponding TFM $R(z)$. Transcribing algebraically the condition $(i)$, we get

$$
R(z) G(z)=0
$$

where

$$
G(z)=\left[\begin{array}{cc}
G_{u}(z) & G_{d}(z) \\
I_{N m_{u}} & O
\end{array}\right],
$$

while the fault detectability condition $(i i)$ requires

$$
R(z) \widetilde{G}_{f}^{(i)}(z) \neq 0, \quad i=1, \ldots, m_{f}
$$

where

$$
\widetilde{G}_{f}^{(i)}(z)=\left[\begin{array}{c}
G_{f}^{(i)}(z) \\
O
\end{array}\right]
$$

From (6) it follows that for the existence of a detector it is necessary that the number of independent measurements is larger than the number of independent disturbances. Moreover, from (8) follows that each fault must act independently from the disturbances on the system.

A necessary and sufficient condition for the existence of a solution to the PFDF is the following one [10]:

Theorem 1: For the periodic system (1) the PFDP is solvable if and only if

$$
\operatorname{rank}\left[G(z) \widetilde{G}_{f}^{(i)}(z)\right]>\operatorname{rank} G(z), \quad i=1, \ldots, m_{f}
$$

An equivalent form of (9) which appears in the works of several authors (see [11] and references therein) is

$$
\operatorname{rank}\left[G_{d}(z) G_{f}^{(i)}(z)\right]>\operatorname{rank} G_{d}(z), i=1, \ldots, m_{f}
$$

In what follows, we prefer to use (9) as basic solvability condition instead of (10) because the proposed computational algorithm can be easily interpreted in terms of condition (9).

A necessary and sufficient condition for the existence of a solution to the PSFDF is:

Theorem 2: For the periodic system (1) the PSFDP is solvable if and only if for each $i=1, \ldots, m_{f}$

$$
\begin{aligned}
& \text { (a) } \operatorname{rank}\left[G(z) \widetilde{G}_{f}^{(i)}(z)\right]>\operatorname{rank} G(z) \\
& \text { (b) }\left[G(z) \widetilde{G}_{f}^{(i)}(z)\right] \text { and } G(z) \text { have the same zeros in } \\
& z=1 .
\end{aligned}
$$

Proof: Additionally to the condition (9) we need to show that there exists a detector $R(z)$ which solves the PSFDF provided the TFM from the fault to the residual signal $R(z) \widetilde{G}_{f}^{(i)}(z)$ has no zero in $z=1$. Since $R(z)$ can be always assimilated with a nullspace basis of $G(z)$, this condition is equivalent to require that $\left[G(z) \widetilde{G}_{f}^{(i)}(z)\right]$ has no additional zeros in $z=1$ as those of $G(z)$. The proof of this assertion follows from the Remark after the proof of Theorem 4 (see Section IV).

Designing a residual filter which solves the PFDP/PSFDP by constructing explicitly the lifted representations is in principe straightforward with the help of methods developed for standard systems (see for example [12] or [13]). Since $R(z)$ must be a left annihilator of $G(z)$, one possibility 
to determine $R(z)$ is to compute first a left minimal basis $N(z)$ for the left nullspace of $G(z)$, and then to build a rational and stable detector as $R(z)=X(z) N(z)$, where $X(z)$ is chosen such that $R(z)$ is stable and the detector fulfills the fault detectability condition $(i i)$ or $\left(i i^{\prime}\right)$. The main difficulty with this approach is that we must ensure for the resulting $R(z)$ to correspond to a lifted causal periodic system which must be realizable in the form (2). Therefore, constructing $N(z)$ and choosing $X(z)$ to correspond to causal periodic realizations, with the additional constraint that the conditions (8) are fulfilled, appears to be non-trivial. Moreover, even in the case when this approach is applicable, severe numerical difficulties are to be expected for systems with large periods and/or orders.

In the next sections, we show that an equivalent approach is possible for periodic systems without resorting to manipulate explicitly lifted representations. The proposed computational approach operates directly on the matrices of the original periodic state-space description (1) and computes left annihilators directly in periodic minimal state-space representations. All subsequent computations to determine a stable detector or to satisfy the detectability constraints are performed on this representation and can be done using reliable numerical techniques based on statespace computations as well.

\section{COMPUTATION OF LEFT ANNiHILATORS}

In this section we propose a computational approach to determine a stable left annihilator for the periodic system

$$
\begin{aligned}
E_{k} x(k+1) & =A_{k} x(k)+B_{k}^{u} u(k)+B_{k}^{d} d(k) \\
{\left[\begin{array}{c}
y(k) \\
u(k)
\end{array}\right] } & =\left[\begin{array}{c}
C_{k} \\
0
\end{array}\right] x(k)+\left[\begin{array}{c}
D_{k}^{u} \\
I_{m_{u}}
\end{array}\right] u(k)+\left[\begin{array}{c}
D_{k}^{d} \\
0
\end{array}\right] d(k)
\end{aligned}
$$

corresponding to the lifted TFM $G(z)$ in (7). In terms of lifted representations, this amounts to determine a periodic system (e.g., of the form (2)), whose lifted TFM $N(z)$ is a proper rational matrix whose columns represent a basis for the left nullspace of $G(z)$ (i.e., $N(z) G(z)=0$ ).

Our method exploits the simple fact [14] that $N(z)$ is a left nullspace basis of $G(z)$ if and only if $[M(z) \mid N(z)]$ is a left nullspace basis of the associated system matrix

$$
S(z)=\left[\begin{array}{ccc}
\widetilde{A}-z \widetilde{E} & \mathcal{B}^{u} & \mathcal{B}^{d} \\
\hline \mathcal{C} & \mathcal{D}^{u} & \mathcal{D}^{d} \\
O & I_{N m_{u}} & O
\end{array}\right]
$$

Thus, to compute $N(z)$ we can determine equivalently a left nullspace basis $Y(z)$ for $S(z)$ and then obtain $N(z)$ as

$$
N(z)=Y(z)\left[\begin{array}{c}
O \\
I_{N\left(p+m_{u}\right)}
\end{array}\right]
$$

Consider now the permuted system matrix $\widetilde{S}(z)=$
$\Pi_{1} S(z) \Pi_{2}$

$$
\widetilde{S}(z)=\left[\begin{array}{ccccc}
S_{1} & -T_{1} & O & \cdots & O \\
O & S_{2} & -T_{2} & \cdots & O \\
\vdots & \ddots & \ddots & \ddots & \vdots \\
O & & & S_{N-1} & -T_{N-1} \\
-z T_{N} & O & \cdots & O & S_{N}
\end{array}\right]
$$

where for $\mathrm{k}=1, \ldots, N$

$$
S_{k}=\left[\begin{array}{ccc}
A_{k} & B_{k}^{u} & B_{k}^{d} \\
C_{k} & D_{k}^{u} & D_{k}^{d} \\
0 & I_{m_{u}} & 0
\end{array}\right], \quad T_{k}=\left[\begin{array}{ccc}
E_{k} & O & O \\
O & O & O \\
O & O & O
\end{array}\right]
$$

It follows that if $\widetilde{Y}(z)$ is a left nullspace basis for $\widetilde{S}(z)$, then $N(z)$ results as

$$
N(z)=\tilde{Y}(z) \widetilde{\mathcal{W}}
$$

where $\widetilde{W}_{k}=\left[\begin{array}{c}O \\ I_{p+m_{u}}\end{array}\right]$.

Let $Q_{k}$ and $Z_{k}$ be orthogonal $N$-periodic matrices determined using the algorithm proposed in [15] to reduce the $N$-periodic pair $\left(S_{k}, T_{k}\right)$ to the Kronecker-like form $\left(\bar{S}_{k}, \bar{T}_{k}\right):=\left(Q_{k} S_{k} Z_{k}, Q_{k} T_{k} Z_{k+1}\right)$, where

$$
\begin{aligned}
& \bar{S}_{k}=\left[\begin{array}{c|ccc}
B_{k}^{r} & A_{k}^{r} & * & * \\
O & O & A_{k}^{r e g} & * \\
O & O & O & A_{k}^{l} \\
\hline O & O & O & C_{k}^{l}
\end{array}\right] \\
& \bar{T}_{k}=\left[\begin{array}{c|ccc}
O & E_{k}^{r} & * & * \\
O & O & E_{k}^{r e g} & * \\
O & O & O & E_{k}^{l} \\
\hline O & O & O & O
\end{array}\right]
\end{aligned}
$$

where: (a) the periodic system $\left(\mathcal{E}^{r}, \mathcal{A}^{r}, \mathcal{B}^{r}, *, *\right)$ is completely reachable and $\mathcal{E}^{r}$ is invertible; (b) the periodic system $\left(\mathcal{E}^{l}, \mathcal{A}^{l}, *, \mathcal{C}^{l}, *\right)$ is completely observable and $\mathcal{E}^{l}$ is invertible; (c) the pole pencil (4) corresponding to the periodic pair $\left(E_{k}^{r e g}, A_{k}^{r e g}\right)$ is regular. Note that the triples $\left(\mathcal{E}^{r}, \mathcal{A}^{r}, \mathcal{B}^{r}\right)$ and $\left(\mathcal{E}^{l}, \mathcal{A}^{l}, \mathcal{C}^{l}\right)$ specify the right and left Kronecker structures of $S(z)$, respectively, while the pair $\left(\mathcal{E}^{r e g}, \mathcal{A}^{r e g}\right)$ specifies the finite and infinite zero structure of $S(z)$.

By exploiting the fine structure of the resulting periodic pair $\left(\bar{S}_{k}, \bar{T}_{k}\right)$, it is possible to bring the pencil $\mathcal{Q} \widetilde{S}(z) \mathcal{Z}$ using appropriate permutation matrices $\Pi_{3}$ and $\Pi_{4}$ in the form $\bar{S}(z)=\Pi_{3} \mathcal{Q} \widetilde{S}(z) \mathcal{Z} \Pi_{4}$

$$
\bar{S}(z)=\left[\begin{array}{c|ccc}
\mathcal{B}^{r} & S^{r}(z) & * & * \\
\mathcal{O} & \mathcal{O} & S^{r e g}(z) & * \\
\mathcal{O} & \mathcal{O} & \mathcal{O} & S^{l}(z) \\
\hline \mathcal{O} & \mathcal{O} & \mathcal{O} & \mathcal{C}^{l}
\end{array}\right]
$$

where, for $x=r, r e g, l$,

$$
S^{x}(z)=\left[\begin{array}{ccccc}
A_{1}^{x} & -E_{1}^{x} & O & \cdots & O \\
O & A_{2}^{x} & -E_{2}^{x} & \cdots & O \\
\vdots & \ddots & \ddots & \ddots & \vdots \\
O & & & A_{N-1}^{x} & -E_{N-1}^{x} \\
-z E_{N}^{x} & O & \cdots & O & E_{N}^{x}
\end{array}\right]
$$


By construction $\left[\begin{array}{lll}\mathcal{B}^{r} S^{r}(z) & * \\ \mathcal{O} & \mathcal{O} & S^{r e g}(z)\end{array}\right]$ has full row rank, excepting possibly a finite set of values of $z$ (i.e., the invariant zeros of $S(z)$ ) and $\left[\begin{array}{c}S^{l}(z) \\ \mathcal{C}^{l}\end{array}\right]$ has full column rank. Thus, we can choose a left nullspace $\bar{Y}(z)$ of $\bar{S}(z)$ in the form

$$
\left.\bar{Y}(z)=\left[\begin{array}{lll}
O & O & -\mathcal{C}^{l}\left[S^{l}(z)\right.
\end{array}\right]^{-1} \quad I\right]
$$

Then the nullspace of $G(z)$ is

$$
N(z)=\bar{Y}(z) \Pi_{3} \mathcal{Q \mathcal { W }}=-\mathcal{C}^{l}\left[S^{l}(z)\right]^{-1} \mathcal{B}^{l}+\mathcal{D}^{l}
$$

where $\mathcal{B}^{l}$ and $\mathcal{D}^{l}$ result from a row partition compatible with (16) of

$$
\Pi_{3} \mathcal{Q} \widetilde{\mathcal{W}}=\left[\begin{array}{l}
\widehat{\mathcal{B}}^{r} \\
\mathcal{B}^{r e g} \\
\mathcal{B}^{l} \\
\hline \mathcal{D}^{l}
\end{array}\right]
$$

Partition now the columns of $B_{k}^{l}$ and $D_{k}^{l}$ conformably to the dimensions of $u(k)$ and $y(k)$, as

$$
B_{k}^{l}=\left[\begin{array}{ll}
H_{k}^{y} & H_{k}^{u}
\end{array}\right], \quad D_{k}^{l}=\left[\begin{array}{ll}
L_{k}^{y} & L_{k}^{u}
\end{array}\right]
$$

Then, a periodic realization for $N(z)$ can be obtained by inspection as

$$
\begin{aligned}
E_{k}^{l} \bar{x}(k+1) & =A_{k}^{l} \bar{x}(k)+H_{k}^{y} y(k)+H_{k}^{u} u(k) \\
\bar{y}(k) & =C_{k}^{l} \bar{x}(k)+L_{k}^{y} y(k)+L_{k}^{u} u(k)
\end{aligned}
$$

Since $\mathcal{E}^{l}$ is nonsingular, this periodic descriptor system can be easily reduced to a standard one as in (2). Note that, this detector is obtained in general with time-varying state and output vector dimensions.

To determine the left annihilator (19), we performed exclusively orthogonal transformations on the system matrices. We can easily prove that all computed matrices are exact for a slightly perturbed original system. It follows that the algorithm to compute the left annihilator is numerically stable.

Proposition 1: The annihilator (19) is minimal.

Proof: By construction, the periodic system $\left(\mathcal{E}^{l}, \mathcal{A}^{l}, \mathcal{B}^{l}, \mathcal{C}^{l}, \mathcal{D}^{l}\right)$ is completely observable, and thus we have to prove only the reachability of this system. Consider the extended system pencil

$$
S_{e}(z)=\left[\begin{array}{ccc|cc}
\widetilde{A}-z \widetilde{E} & \mathcal{B}^{u} & \mathcal{B}^{d} & O & O \\
\hline \mathcal{C} & \mathcal{D}^{u} & \mathcal{D}^{d} & I_{N p} & O \\
O & I_{N m_{u}} & O & O & I_{N m_{u}}
\end{array}\right]
$$

This matrix has full row rank, excepting those values of $z$ which belong to the unreachable eigenvalues of the pair $\left(\widetilde{A}-z \widetilde{E},\left[\mathcal{B}^{u} \mathcal{B}^{d}\right]\right)$ (called also the input decoupling zeros of the periodic system [16]). Since these eigenvalues appears in the subpencil $S_{r e g}(z)$ (being part of the invariant zeros of $S(z)$ ), the subpencil formed from the the last two block rows of the transformed extended pencil

$$
\Pi_{3} \mathcal{Q} \Pi_{1} S_{e}(z)\left[\begin{array}{cc}
\Pi_{2} \mathcal{Z} \Pi_{4} & O \\
O & I_{N\left(p+m_{u}\right)}
\end{array}\right]=
$$

$$
\left[\begin{array}{cccc|l}
\mathcal{B}^{r} & S^{r}(z) & * & * & \widehat{\mathcal{B}}^{r} \\
\mathcal{O} & \mathcal{O} & S^{r e g}(z) & * & \mathcal{B}^{r e g} \\
\mathcal{O} & \mathcal{O} & \mathcal{O} & S^{l}(z) & \mathcal{B}^{l} \\
\hline \mathcal{O} & \mathcal{O} & \mathcal{O} & \mathcal{C}^{l} & \mathcal{D}^{l}
\end{array}\right]
$$

has full row rank. Therefore, we have that the subpencil $\left[\begin{array}{lll}S^{l}(z) & \mathcal{B}^{l}\end{array}\right]$ has full row rank as well, and according to [5], the periodic system $\left(\mathcal{E}^{l}, \mathcal{A}^{l}, \mathcal{B}^{l}, \mathcal{C}^{l}, \mathcal{D}^{l}\right)$ is completely reachable.

The resulted left annihilator in (19) is in general not stable (i.e., some characteristic multipliers of the periodic matrix $\left[E_{k}^{l}\right]^{-1} A_{k}^{l}$ may have moduli greater or equal to one). To compute a stable left annihilator, we can perform an additional transformation on the reduced periodic pair $\left(\bar{S}_{k}, \bar{T}_{k}\right)$ using the transformation matrix $U_{k}$ of the form

$$
U_{k}=\left[\begin{array}{ccc|c}
I & 0 & 0 & 0 \\
0 & I & 0 & 0 \\
0 & 0 & I & K_{k} \\
\hline 0 & 0 & 0 & I
\end{array}\right]
$$

where the identity matrices have dimensions compatible with the block row structure of the reduced matrices $\bar{S}_{k}$ in (14). Then the transformed pair $\left(\widehat{S}_{k}, \widehat{T}_{k}\right):=\left(U_{k} \bar{S}_{k}, U_{k} \bar{T}_{k}\right)$ is given by

$$
\widehat{S}_{k}=\left[\begin{array}{c|ccc}
B_{k}^{r} & A_{k}^{r} & * & * \\
O & O & A_{k}^{r e g} & * \\
O & O & O & A_{k}^{l}+K_{k} C_{k}^{l} \\
\hline O & O & O & C_{k}^{l}
\end{array}\right], \quad \widehat{T}_{k}=\bar{T}_{k}
$$

The periodic system representing the corresponding stable annihilator is defined by

$$
\begin{aligned}
E_{k}^{l} \bar{x}(k+1) & =\bar{A}_{k}^{l} \bar{x}(k)+\bar{H}_{k}^{u} u(k)+\bar{H}_{k}^{y} y(k) \\
\bar{y}(k) & =C_{k}^{l} \bar{x}(k)+L_{k}^{u} u(k)+L_{k}^{y} y(k)
\end{aligned}
$$

where

$$
\begin{aligned}
\bar{A}_{k}^{l} & =A_{k}^{l}+K_{k} C_{k}^{l} \\
\bar{H}_{k}^{u} & =H_{k}^{u}+K_{k} L_{k}^{u} \\
\bar{H}_{k}^{y} & =H_{k}^{u}+K_{k} L_{k}^{y}
\end{aligned}
$$

To obtain annihilators with the poles (characteristic multipliers) lying in a "good" domain $\mathbb{C}_{g}$ of the complex plane (e.g., interior of the unit circle), we can solve a periodic stabilization or pole assignment problem. Note that the poles of the detector are the eigenvalues of the matrix $\left(E_{N}^{l}\right)^{-1}\left(A_{N}^{l}+K_{N} C_{N}^{l}\right) \cdots\left(E_{1}^{l}\right)^{-1}\left(A_{1}^{l}+K_{1} C_{1}^{l}\right)$. Therefore, by choosing appropriate $K_{k}$ all poles can be moved to arbitrary locations in $\mathbb{C}_{g}$. This is guaranteed by the observability of the periodic system $\left(\mathcal{E}^{l}, \mathcal{A}^{l}, *, \mathcal{C}^{l}, *\right)$.

\section{DeteCtor Design ISSUES}

\section{A. Checking fault detectability}

Until now we focussed on requirement (i) by providing a new numerical algorithm for computing a stable left annihilator of the extended system (11). To be useful as a residual generator, the computed annihilator must also satisfy the requirement (ii) to generate non-zero residual signals in the case of faults occuring in the system. This 
requirement is implicitly contained in condition (9) for the existence of a solution of the PFDP.

We will show that condition (9) can be simply checked from the results obtained by reducing the matrices of the periodic pair $\left(S_{k}, T_{k}\right)$ to the Kronecker-like forms $\bar{S}_{k}$ and $\bar{T}_{k}$ in (14) and (15), respectively. For the resulting periodic orthogonal transformation matrix $Q_{k}$, we compute for each column index $i$ and for $k=1, \ldots, N$

$$
S_{k}^{f, i}:=Q_{k}\left[\begin{array}{c}
B_{k}^{f, i} \\
D_{k}^{f, i} \\
O
\end{array}\right]=\left[\begin{array}{c}
* \\
* \\
\widetilde{B}_{k}^{f, i} \\
\hline \widetilde{D}_{k}^{f, i}
\end{array}\right]
$$

where the row partitioning of the rightmost matrix above is compatible with the row partitioning of $\bar{S}_{k}$ in (14). Using the row permutation $\Pi_{3}$ used to get $\bar{S}(z)$ in (16), we obtain

$$
\Pi_{3} \mathcal{S}^{f, i}=\left[\begin{array}{c}
* \\
* \\
\widetilde{\mathcal{B}}^{f, i} \\
\widetilde{\mathcal{D}}^{f, i}
\end{array}\right]
$$

The condition (9) for the existence of a solution to the PFDP can be expressed as follows:

Theorem 3: For the periodic system (1) the PFDP is solvable if and only if

$$
\left[\begin{array}{c}
\widetilde{\mathcal{B}}^{f, i} \\
\widetilde{\mathcal{D}}^{f, i}
\end{array}\right] \neq 0, \quad i=1, \ldots, m_{f}
$$

Proof: For the detector $R(z)=\bar{Y}(z)$ in (17), condition (9) is equivalent to (8), which can be expressed as

$G_{r f}^{(i)}(z):=\bar{Y}(z)\left[\begin{array}{c}* \\ * \\ \widetilde{\mathcal{B}}^{f, i} \\ \widetilde{\mathcal{D}}^{f, i}\end{array}\right]=-\mathcal{C}^{l}\left[S^{l}(z)\right]^{-1} \widetilde{\mathcal{B}}^{f, i}+\widetilde{\mathcal{D}}^{f, i} \neq 0$

where $G_{r f}^{(i)}(z)$ is the lifted TFM from the fault signal $f_{i}$ to the residual signal $r$. Since the corresponding periodic system realization $\left(\mathcal{E}^{l}, \mathcal{A}^{l}, \widetilde{\mathcal{B}}^{f, i}, \mathcal{C}^{l}, \widetilde{\mathcal{D}}^{f, i}\right)$ is completely observable, the condition that $G_{r f}^{(i)}(z)$ is nonzero is (22).

To check the existence of a detector, we have to verify only condition (22). Since the orthogonal matrix $\mathcal{Q}$ is always accumulated, this check involves practically no additional computations and can be seen as part of the design procedure. Similar checks can be used to define different fault isolation schemes (see for example [1], [9]).

Theorem 4: For the periodic system (1) the PSFDP is solvable if and only if the periodic system $\left(\mathcal{E}^{l}, \mathcal{A}^{l}, \widetilde{\mathcal{B}}^{f, i}, \mathcal{C}^{l}, \widetilde{\mathcal{D}}^{f, i}\right)$ has no zeros in $z=1$.

Proof: A fault detector which solves the PSFDP ensures that $z=1$ is not a zero of $G_{r f}^{(i)}(z)$ (otherwise an asymptotically vanishing residual signal results for a constant nonzero fault signal). Equivalently, this condition says that $z=1$ is not a zero of the periodic system $\left(\mathcal{E}^{l}, \mathcal{A}^{l}, \widetilde{\mathcal{B}}^{f, i}, \mathcal{C}^{l}, \widetilde{\mathcal{D}}^{f, i}\right)$.
Remark: The zeros of this system are the additional zeros to those of $G(z)$ which result when forming the realization of $\left[G(z) \widetilde{G}_{f}^{(i)}(z)\right]$. Therefore, the above condition is equivalent to the conditions of Theorem 2 .

To perform this check, we can compute the zeros of the periodic system $\left(\mathcal{E}^{l}, \mathcal{A}^{l}, \widetilde{\mathcal{B}}^{f, i}, \mathcal{C}^{l}, \widetilde{\mathcal{D}}^{f, i}\right)$ using the algorithm of [17]. In the case when the annihilator is stable (or has been already stabilized), a simpler test is to evaluate $G_{r f}^{(i)}(1)$ (i.e., the steady state gain). This can be done using an efficient algorithm to compute gains as that one of [18].

\section{B. Solving fault isolation problems}

Fault isolation requires a complete decoupling of faults to ensure that each residual signal $r_{i}(k)$ is influenced only by the corresponding fault $f_{i}(k)$. Thus the periodic fault detection and isolation problem (PFDIP) can be formulated as follows:

Periodic Fault Detection and Isolation Problem: Determine a periodic linear residual generator having the general form (2) such that

(i) $r(k)=0$ if $f(k)=0$ (fault-free case);

(ii) $r_{i}(k) \neq 0$ if $f_{i}(k) \neq 0$, for $i=1, \ldots, m_{f}$ (fault isolability),

where for both (i) and (ii) we assume zero initial conditions for the state vectors.

A possible approach to solve the PFDIP is to design a bank of $m_{f}$ detectors, each of form (2), such that each detector is sensible only to one fault and insensible to the rest of faults. To achieve this goal we can formally redefine the rest of faults as disturbances and solve a standard PFDP for each fault. In this way, the resulting bank of detectors behaves as a fault detection and isolation filter which solves the PFDIP.

To formalize this approach, let partition and permute the columns of $B_{k}^{f}$ and $D_{k}^{f}$ compatibly as

$$
B_{k}^{f}=\left[B_{k}^{f, i} B_{k}^{f, \bar{i}}\right] \quad B_{k}^{f}=\left[B_{k}^{f, i} B_{k}^{f, \bar{i}}\right]
$$

where $B_{k}^{f, i}$ and $D_{k}^{f, i}$ are the $i$-th columns of matrices $B_{k}^{f}$ and $D_{k}^{f}$, respectively, and $B_{k}^{f, \bar{i}}$ and $D_{k}^{f, \bar{i}}$ are the rest of columns in these matrices. Let denote by $G_{f}^{(i)}(z)$ and $G_{f}^{(\bar{i})}(z)$ the TFMs of the lifted systems corresponding to the periodic systems $\left(\mathcal{E}, \mathcal{A}, \mathcal{B}^{f, i}, \mathcal{C}, \mathcal{D}^{f, i}\right)$ and $\left(\mathcal{E}, \mathcal{A}, \mathcal{B}^{f, i}, \mathcal{C}, \mathcal{D}^{f, i}\right)$, respectively. Define

$$
\widetilde{G}_{f}^{(i)}(z)=\left[\begin{array}{c}
G_{f}^{(i)}(z) \\
O
\end{array}\right], \quad \widetilde{G}_{f}^{(\bar{i})}(z)=\left[\begin{array}{c}
G_{f}^{(\bar{i})}(z) \\
O
\end{array}\right]
$$

The PFDIP can be solved if each fault can be completely decoupled from the rest of faults, and therefore we can state immediately the following solvability condition:

Theorem 5: For the periodic system (1) the PFDIP is solvable if and only if for each $i=1, \ldots, m_{f}$

$$
\operatorname{rank}\left[G(z) \widetilde{G}_{f}^{(\bar{i})}(z) \widetilde{G}_{f}^{(i)}(z)\right]>\operatorname{rank}\left[G(z) \widetilde{G}_{f}^{(\bar{i})}(z)\right]
$$

This theorem is the TFM based equivalent formulation of Theorem 3 in [19]. To check fault isolability, conditions similar to (22) can be checked. 


\section{Computing approximate detectors}

The detectors have generically orders which are comparable to the orders of the underlying applications. Besides exact order reduction (e.g., using covers based techniques [13]), it is possible to try to use reduced order detector approximations computed via model reduction techniques. Since the resulting truncation error can be exactly evaluated, such reduced order detectors can be useful provided appropriate thresholds reflecting this error are employed. A simple way to reduce the order of a detector with the lifted TFM $R(z)$ is to determine a reduced order detector with the lifted TFM $\widetilde{R}(z)$ by solving the frequency-weighted model reduction problem

$$
\|(R(z)-\widetilde{R}(z)) G(z)\|_{\infty}=\min
$$

Note that $R(z)$ has been designed such that $R(z) G(z)=0$, and therefore using $G(z)$ as frequency weighting appears a natural choice to force that $\widetilde{R}(z) G(z) \approx 0$.

To solve the above approximation problem, the squareroot and balancing-free method for frequency-weighted balanced truncation [20] can be extended to periodic systems along the lines of the algorithm described in [21]. Interestingly, any such projection based approach will not find the trivial global solution $\widetilde{R}(z)=0$, but rather will try to find a solution which approximates the original detector.

\section{CONCLUSiOnS}

We proposed a numerically sound computational approach to design fault detectors for periodic systems. The main computational ingredient of the proposed approach is the computation of a left periodic annihilator of a certain periodic system. To compute such an annihilator a numerically stable algorithm based on orthogonal structure preserving pencil reduction has been proposed. The main advantage of our approach over other possible techniques is that the computation of the annihilator can entirely be done by manipulating state space matrices of the original periodic system (instead manipulating those of lifted time-invariant models). The resulting annihilator is obtained directly in a periodic minimal system representation. The proposed approach is also applicable to descriptor periodic systems with singular or even rectangular $E_{k}$.

An important problem which has not been addressed is the computation of least order detectors. We believe that this problem can be tackled using extensions to the periodic case of the minimal dynamic covers techniques employed in [13]. The computational problem of determining minimal order dynamic covers for standard state space systems has been recently addressed in [22]. The proposed computational algorithm is essentially a modified staircase reachability form computation as that proposed in [23]. A similar algorithm for periodic systems has been proposed recently [24], and this algorithm could serve as basis to develop a similar cover design algorithm for periodic systems.

\section{ACKNOWLEDGMENTS}

The work of the author has been performed in the framework of the Swedish Strategic Research Foundation Grant "Matrix Pencil Computations in Computer-Aided Control System Design: Theory, Algorithms and Software Tools".

\section{REFERENCES}

[1] J. Gertler, Fault Detection and Diagnosis in Engineering Systems. New York: Marcel Dekker, 1998.

[2] M. S. Fadali and H. E. Emara-Shabaik, "Timely robust fault detection for multirate linear systems," Int. J. Control, vol. 75, pp. 305-313, 2002.

[3] P. Zhang, S. X. Ding, and G. Z. W. uand D. Z. Zhou, "Fault detection for multirate sampled-data systems with time delay," Int. J. Control, vol. 75 , p. 14571471, 2002.

[4] R. A. Meyer and C. S. Burrus, "A unified analysis of multirate and periodically time-varying digital filters," IEEE Trans. Circuits Syst., vol. 22, pp. 162-168, 1975.

[5] O. M. Grasselli and S. Longhi, "Finite zero structure of linear periodic discrete-time systems," Int. J. Systems Sci., vol. 22, pp. 1785-1806, 1991.

[6] A. Varga, "Computation of minimal periodic realizations of transferfunction matrices," IEEE Trans. Automat. Control, vol. 46, pp. 146149, 2004.

[7] P. Zhang, S. X. Ding, G. Z. Wang, and D. H. Zhou, "Fault detection for linear discrete-time periodic systemss," Proc. of IFAC Symp. SAFEPROCESS 2003, Washington, D.C., USA, 2003, pp. 247-252.

[8] A. Varga, "New computational approach for the design of fault detection and isolation filters," in Advances in Automatic Control, ser. The Kluwer International Series in Engineering and Computer Science, M. Voicu, Ed., vol. 754. Kluwer Academic Publishers, 2003, pp. 367-381.

[9] J. Chen and R. J. Patton, Robust Model-Based Fault Diagnosis for Dynamic Systems. Kluwer Academic Publishers, London, 1999.

[10] X. Ding and P. M. Frank, "Frequency domain approach and threshold selector for robust model-based fault detection and isolation," Proc. of IFAC Symp. SAFEPROCESS 1991, Baden-Baden, Germany, 1991.

[11] M. Nyberg, "Criterions for detectability and strong detectability of faults in linear systems," Int. J. Control, vol. 75, pp. 490-501, 2002.

[12] E. Frisk and M. Nyberg, "A minimal polynomial basis solution to residual generation for fault diagnosis in linear systems," Automatica, vol. 37, pp. 1417-1424, 2001.

[13] A. Varga, "On computing least order fault detectors using rational nullspace bases," Proc. of IFAC Symp. SAFEPROCESS'2003, Washington D.C., 2003.

[14] G. Verghese, P. Van Dooren, and T. Kailath, "Properties of the system matrix of a generalized state-space system," Int. J. Control, vol. 30, pp. 235-243, 1979.

[15] A. Varga, "Computation of Kronecker-like forms of periodic matrix pairs," Proc. of MTNS'04, Leuven, Belgium, 2004.

[16] - "Computation of generalized inverses of periodic systems," Proc. of CDC'04, Paradise Island, Bahamas, 2004.

[17] — "Stronly stable algorithms for computing periodic system zeros," Proc. of CDC'2003, Maui, Hawaii, 2003.

[18] — - "Computation of transfer functions matrices of periodic systems," Int. J. Control, vol. 76, pp. 1712-1723, 2003.

[19] M. Hou and P. C. Müller, "A correspondence about FDI observers," Int. J. Control, vol. 76, pp. 295-298, 2003.

[20] A. Varga and B. D. O. Anderson, "Accuracy-enhancing methods for balancing-related frequency-weighted model and controller reduction," Automatica, vol. 39, pp. 919-927, 2003.

[21] A. Varga, "Balanced truncation model reduction of periodic systems," Proc. CDC'2000, Sydney, Australia, 2000, pp. 2379-2384.

[22] — "Reliable algorithms for computing minimal dynamic covers," Proc. of CDC'2003, Maui, Hawaii, 2003.

[23] — - "Numerically stable algorithm for standard controllability form determination," Electron. Lett., vol. 17, pp. 74-75, 1981.

[24] —_ "Computation of Kalman decompositions of periodic systems," European Journal of Control, vol. 10, 2004. 